\title{
Estresse e Características Resilientes em Alunos com Deficiência e TFE na UFPA
}

\author{
Rosamaria Reo Pereira ${ }^{1}$ \\ ${ }^{1}$ Universidade Federal do Pará, PA, Brasil. \\ Rosana Assef Faciola ${ }^{1}$ \\ ${ }^{1}$ Universidade Federal do Pará, PA, Brasil. \\ Maély Ferreira Holanda Ramos ${ }^{1}$ \\ ${ }^{1}$ Universidade Federal do Pará, PA, Brasil.
}

\author{
Simone Souza da Costa Silva ${ }^{1}$ \\ ${ }^{1}$ Universidade Federal do Pará, PA, Brasil. \\ Fernando Augusto Ramos Pontes ${ }^{1}$ \\ ${ }^{1}$ Universidade Federal do Pará, PA, Brasil. \\ Edson Marcos Leal Soares Ramos ${ }^{1}$ \\ ${ }^{1}$ Universidade Federal do Pará, PA, Brasil.
}

Resumo: Poucos são os pesquisadores que tratam da presença do estresse e da resiliência em alunos com deficiência e com Transtornos Funcionais Específicos no ensino superior, o que sinaliza a necessidade dessas duas temáticas serem mais estudadas e melhor compreendidas no ambiente universitário. A presente pesquisa visou descrever as características sociodemográficas e acadêmicas de 50 estudantes universitários participantes da pesquisa e correlacioná-las com os níveis de estresse e de resiliência. Os instrumentos utilizados foram um questionário semiestruturado e duas escalas: Escala de Estresse Percebido e Escala de Resiliência. Os resultados foram analisados por meio da estatística descritiva e da técnica estatística exploratória. Os resultados apontaram que alunos com níveis elevados de estresse têm níveis moderados e baixos de resiliência. A partir desse resultado, concluiu-se a necessidade de desenvolvimento de programas que visem à manutenção de estratégias eficazes de enfrentamento diante de situações adversas dentro do contexto acadêmico. Espera-se que este estudo possa contribuir com a construção de políticas institucionais que favoreça a inclusão de fato de estudantes com deficiência e com TFE na Universidade Federal do Pará e em outras instituições de ensino superior.

Palavras-chave: Estresse, Resiliência, Estudante com Deficiência, Transtorno Funcional Específico, Ensino Superior.

\section{Stress and Resilience Characteristics of Students with Disability and with Specific Functional Disorders at UFPA}

\begin{abstract}
There are few studies dealing with the presence of stress and resilience on undergraduate students with disabilities and with specific functional disorders in higher education, which indicates the need for these two themes to be more studied and better understood in the university environment. The present study aimed to describe the sociodemographic and academic characteristics of 50 university students who participated in the study and correlate them with levels of stress and resilience. The instruments used were a semi-structured questionnaire and two scales: Perceived Stress Scale and Resilience Scale. The results were analyzed through descriptive statistics and the exploratory statistical technique. The results showed that students with high levels of stress have moderate and low levels of resilience. From this result, it was concluded the need to develop programs that aim at the maintenance of effective coping strategies in adverse situations within the academic context. It is expected that this study may contribute to the construction of institutional policies that favor the inclusion in fact of students with disabilities and with specific functional disorders at the Federal University of Pará and other higher education institutions.
\end{abstract}

Keywords: Stress, Resilience, Students with Disabilities, Specific Functional Disorder, Higher Education. 


\title{
Estrés y Características Resilientes en Alumnos con Discapacidad y TFE en la UFPA
}

\begin{abstract}
Resumen: Pocos son los investigadores que tratan de la presencia del estrés y de la resiliencia en alumnos con discapacidad y con trastornos funcionales específicos en la enseñanza superior, lo que señala la necesidad de que estas dos temáticas sean más estudiadas y mejor comprendidas en el ambiente universitario. La presente investigación apunta a describir las características sociodemográficas y académicas de 50 estudiantes universitarios participantes de la investigación y correlacionarlas con los niveles de estrés y de resiliencia. Los instrumentos utilizados fueron un cuestionario semiestructurado y dos escalas: Escala de estrés percibido y Escala de Resiliencia. Los resultados se analizaron mediante la estadística descriptiva y la técnica estadística exploratoria. Los resultados apuntaron que los alumnos con niveles elevados de estrés tienen niveles moderados y bajos de resiliencia. A partir de ese resultado, se concluyó la necesidad de desarrollar programas que apunten al mantenimiento de estrategias eficaces de enfrentamiento ante situaciones adversas dentro del contexto académico. Se espera que este estudio pueda contribuir con la construcción de políticas institucionales que favorezca la inclusión de hecho de estudiantes con discapacidad en la Universidad Federal de Pará y en otras instituciones de enseñanza superior. Los resultados fueron analizados por medio de la estadística descriptiva y de la técnica estadística exploratoria.
\end{abstract}

Palabras clave: Estrés, Resiliencia, Estudiante con Discapacidad, Trastorno Funcional Específico, Enseñanza Superior.

A proposta da educação inclusiva propõe que as instituições de ensino tanto básico quanto superior se preparem para receber as pessoas com deficiência. No entanto, um dos impedimentos encontrados para colocar em prática essa inclusão está ligado ao preconceito e à falta de conhecimento que a sociedade em geral tem sobre a diversidade, e sobre as maneiras de se realizar a inclusão, levando muitas vezes a exclusão da sociedade (Barroco, 2003).

Ao tratar da educação inclusiva, Sanchez (2005) afirma que a mesma objetiva apoiar as qualidades e necessidades de cada um e de todos os estudantes. $\mathrm{O}$ autor sustenta a necessidade de se "pensar na heterogeneidade do alunado como uma questão normal do grupo/classe e pôr em macha um delineamento educativo que permita aos docentes utilizar os diferentes níveis instrumentais e atitudinais como recursos intrapessoais e interpessoais que beneficiem todos os alunos" (p. 12).

No que diz respeito ao ensino superior, o acesso de alunos com deficiência a esse nível de ensino é um direito adquirido por lei e cabe à instituição dispor de adaptações necessárias para que o mesmo possa permanecer e concluir o curso. Neste sentido, a Política
Nacional de Educação Especial na Perspectiva da Educação Inclusiva evidencia que o objetivo da educação especial no âmbito do ensino superior é promover ações que permitam o acesso, a permanência e a participação dos estudantes com deficiência no ensino superior. No entanto, deve haver o planejamento de recursos e serviços para a viabilização da acessibilidade arquitetônica, das comunicações, dos sistemas de informação e dos materiais didáticos e pedagógicos. Todos esses serviços devem ser disponibilizados desde os processos seletivos até o desenvolvimento de todas as atividades que envolvam o ensino, a pesquisa e a extensão (Brasil, 2008).

Ao ingressar no ensino superior, os estudantes enfrentam novos desafios, pois este momento configura-se como um período de transição em que irão precisar se adaptar a um novo estilo de vida, assim como, enfrentar a complexidade do curso escolhido. Este momento quando não bem administrados pode prejudicar a vida social e pessoal, bem como sua trajetória acadêmica, podendo gerar estresse. Neste sentido, vários fatores podem contribuir para o desencadeamento do estresse como: quantidades excessivas de informações em pequeno intervalo de 
tempo, a adaptação cotidiana às novas tecnologias, o elevado custo da educação e a falta de tempo de conciliar a vida acadêmica com as atividades sociais (Baptista, \& Campos, 2000).

\section{Estresse}

Estresse, para Mota-Cardoso, Araujo, Ramos, Gonçalves e Ramos (2002), vem ser um desequilíbrio entre o que o ambiente exige do indivíduo e os recursos de que esta pessoa dispõe para se adaptar as exigências do contexto. Quando o problema requer esforços além do que a pessoa dispõe, torna-se ameaçador, e assim, altera o equilíbrio estando, portanto, diante de uma situação estressante. Por outro lado, o estresse tem a função também de motivar, de servir como energia para uma pessoa em busca de suas realizações, que podem ser sentimentais ou profissionais. Na ausência do estresse, o indivíduo se torna acomodado e desesperado para os desafios diários e geralmente apresentam baixo autoestima. Porém em excesso os efeitos do estresse são mais prejudiciais à saúde (Lipp, 2004).

Para Mondardo e Pedon (2005), o estudante ao ingressar no ensino superior assumirá atividades de alto desempenho, exigindo do mesmo a concentração de esforços. A rotina de estudos constantes e crescente pode se tornar um fator potencialmente estressor, pois a vida acadêmica representa um aumento de responsabilidade, ansiedade e competitividade.

\section{Resiliência}

Por resiliência entende-se o conjunto de processos sociais e intrapsíquicos que possibilitam o desenvolvimento saudável do indivíduo, mesmo tendo este vivenciado experiências desfavoráveis. A complexidade de constructo envolve a interação entre eventos de vida adversos e fatores de proteção, internos e externos ao indivíduo (Pesce, Assis, Avanci, Malaquias, \& Oliveira, 2005).

A esse respeito, Vianna (2008) alega que há controvérsias sobre a definição de resiliência como um atributo individual ou fruto da interação com o ambiente, pois alguns estudiosos a definiram como uma característica estável, uma qualidade individual presente no indivíduo para melhor lidar com eventos hostis na vida. Já outros pesquisadores argumentam que o contexto social pode influenciar nas atitudes resilientes, uma vez que a resiliência está relacionada às competências individuais e interações relacionais.

Silva e Motta (2009) salientam que as investigações que dizem respeito à resiliência têm mudado a forma de perceber o homem, pois sai de um modelo de risco baseado nas necessidades e na doença para um modelo de prevenção e promoção que se baseia nas potencialidades da pessoa. A resiliência, para $o$ autor, é efetiva não apenas para enfrentar adversidades presentes no dia a dia, mas também para a promoção da saúde mental e emocional, pois pode reduzir a intensidade do estresse e sinais emocionais negativos, como depressão, raiva ou ansiedade.

Nesta perspectiva, estudantes com deficiência poderão ter mais dificuldade para ultrapassar os desafios, especialmente quando seu ambiente não constitui um elemento facilitador desta tarefa. Esses alunos podem enfrentar barreiras ao chegar à universidade, por isso é necessário que a mesma possa minimizar esses problemas e promover condições para que todos participem democraticamente do processo de ensino-aprendizagem, sem prejuízo nenhum para a sua vida pessoal e acadêmica (Tavares, 2002).

Poucos são os pesquisadores que tratam da presença do estresse e da resiliência em alunos com deficiência no ensino superior, o que sinaliza a necessidade dessas duas temáticas serem mais estudadas e melhor compreendidas no ambiente universitário. O ingresso do estudante com deficiência no ensino superior vem chamando a atenção de estudiosos de diversas áreas de conhecimento. As pesquisas são importantes no sentido de contribuir para o processo de integração desse estudante à vida universitária, já que trazem informações relevantes sobre as dificuldades com as quais esses estudantes se deparam neste ambiente. Assim, tendo em vista a importância dos estudos sobre esses dois temas, a presente pesquisa visou descrever as características sociodemográficas e acadêmicas de estudantes com deficiência e com transtornos funcionais específicos da Universidade Federal do Pará e correlacioná-las com os níveis de estresse e de resiliência.

\section{Método}

\section{Participantes}

Para realização da pesquisa, a amostra foi por conveniência, constituída de 192 alunos, sendo esta- 
belecidos os seguintes critérios de inclusão: ser estudante com deficiência e com transtornos funcionais específicos da graduação, estar regularmente matriculado entre os anos de 2012 a 2015, estudar no campus do Guamá e ter dificuldades na realização de suas atividades acadêmicas.

\section{Procedimento de seleção dos participantes}

Inicialmente, foi feito contato com a Direção Geral do Centro de Registros e Indicadores Acadêmicos (CIAC) e com a Coordenadoria de Acessibilidade (CoAcess) da UFPA sendo solicitadas informações referentes aos estudantes matriculados na instituição no período de 2012 a 2015. Após apreciação do projeto, estas unidades liberaram uma lista com o nome de 192 estudantes, assim como o curso, o ano de matrícula, o tipo de deficiência ou transtorno, o contato telefônico e o e-mail. Dos 192 estudantes que foram contactados, 142 foram excluídos da pesquisa pelos seguintes motivos: um estudante não estudava no campus do Guamá, 66 alunos afirmaram que a sua deficiência não lhes prejudicava dentro do campus e que conseguiam desenvolver suas atividades acadêmicas sem nenhuma dificuldade, 43 apresentaram problemas com o contato disponibilizado, 22 cancelaram o curso e dez estudantes não aceitaram participar do estudo por diversos motivos particulares. 50 estudantes aceitaram participar do estudo.

A coleta de dados ocorreu entre os meses de janeiro a julho de 2016 nas dependências físicas da universidade, em horário preestabelecido pelos participantes e que não interferiu nas atividades acadêmicas dos mesmos. No início da entrevista, cada participante foi informado dos objetivos gerais do estudo, formalizado o convite para participação e assinado o Termo de Consentimento Livre Esclarecido (TCLE), garantindo a participação voluntária, a confidencialidade e o anonimato destes alunos. O preenchimento do questionário demorou aproximadamente 60 minutos. As entrevistas foram realizadas pela própria pesquisadora que preencheu o questionário de forma individualizada. As respostas foram transcritas de forma literal.
Os 50 estudantes foram submetidos à Escala de Estresse Percebido (Luft et al, 2007) e após somatória das pontuações das 14 questões a pontuação dos participantes analisados variou entre a mínima de 11 e a máxima de 49. A média estabelecida de 27 foi o ponto de corte estabelecido para dividir os 50 participantes em dois grupos. 21 estudantes obtiveram escores abaixo ou igual à média foram classificados com baixo nível de estresse e 29 participantes que alcançaram escores acima da média foram classificados com alto nível de estresse.

\section{Instrumentos de coleta}

O questionário sociodemográfico e acadêmico foi adaptado do questionário socioeconômico ${ }^{1}$ do Instituto Federal de Educação, Ciências e Tecnologia do Pará - campus Belém pela pesquisadora e buscou conhecer o perfil dos estudantes participantes da pesquisa que estudavam no campus do Guamá. Constou de questões referentes ao levantamento de variáveis sociodemográficas e acadêmicas.

A Escala de Estresse Percebido (EEP) foi formulada originalmente por Cohen Karmack, \& Mermelsteinm (1983) traduzida e validada para o Brasil por Luft et al. (2007). A EEP possui 14 questões com opções de resposta que variam de zero a quatro $(0=$ nunca; 1 = quase nunca; 2 = às vezes; 3 = quase sempre; 4 = sempre). $\mathrm{O}$ total da escala é a soma das pontuações destas 14 questões e os escores podem variar de zero a 56 .

A resiliência foi mensurada por meio da Escala de Resiliência desenvolvida por Wagnild e Young (1993) e validada e adaptada para o Brasil por Pesce et al. (2005). Possui 25 itens, descritos de forma positiva com resposta tipo Likert variando de 1 (discordo totalmente) a 7 (concordo totalmente). Os escores da escala oscilam entre 25 a 175 pontos. Esta escala possui três fatores: o Fator 1 denominado Competência Pessoal e Aceitação de si e da vida. O Fator 2 diz respeito à Independência e Determinação. O Fator 3 refere-se à Autoconfiança e Capacidade de adaptação a situações da vida (Pesce et al., 2005).

\section{Procedimentos éticos}

O estudo foi submetido ao Comitê de Ética em Pesquisa com Seres Humanos do Instituto de

\footnotetext{
${ }^{1}$ Recuperado de http://belem.ifpa.edu.br/documentos/dcom/28-prosel-ifpa-belem-2015-anexo-v-questionario-socioeconomico/file
} 
Ciências da Saúde da Universidade Federal do Pará (CEP-ICS/UFPA), tendo sido aprovado na reunião do dia 3 de novembro de 2014 (CAAE: 36145314.9.0000.0018 e Parecer $n^{\circ}$ 855.169).

\section{Procedimento de análise}

Os dados coletados foram catalogados e arquivados em base de dados construída no programa Statistical Package for the Social Sciences (SPSS), versão 20 para Windows. Para verificar a associação das categorias das variáveis quantitativas realizou-se a Análise de Correspondência (AC). A AC, segundo Fávero, Belfiori, Silva e Chan (2009), é uma técnica estatística exploratória utilizada para verificar associações ou similaridades entre variáveis qualitativas ou variáveis contínuas categorizadas.

A AC foi realizada com o auxílio do aplicativo Statistica, versão 6.0. Em todos os testes, fixou-se $\alpha=5 \%(p \leq 0,05)$ para rejeição da hipótese nula. As variáveis foram categorizadas da seguinte maneira: ( $i$ ) Sexo: Masculino e Feminino; (ii) Área de Conhecimento: Filosofia e Ciências Humanas; Ciências da Saúde; Ciências Sociais e Aplicadas e Ciências Jurídicas e Ciências Exatas e Naturais; (iii) Deficiência: Mobilidade Reduzida e Cadeirante, Baixa Visão ou Cegueira; Deficiência Auditiva e Surdez e Outras; (iv) Faixa Etária: 18 a 21 Anos; 22 a 28 Anos e 29 a 45 Anos; $(v)$ Resiliência: Baixa (89 a 135), Moderada (136 a 153) e Alta (154 a 166); ( $\nu$ ) Estresse: Baixo $(\leq 27)$ eAlto (>27); ( vi) Fator 1 da Escala de Resiliência: Baixo (0 a 40,51), Moderado $(40,52$ a 78,63) e Alto $(78,63$ a 100); (vi) Fator 2 da Escala de Resiliência: Baixo (0 a $50,60)$, Moderado $(50,61$ a 93,29$)$ e Alto $(93,30$ a 100$)$; ( vi) Fator 3 da Escala de Resiliência: Baixo (0 a 33,08), Moderado (33,09 a 71,51) e Alto (71,52 a 100).

\section{Resultados}

Nesta parte serão apresentados os resultados desta pesquisa, inicialmente caracterizando os estudantes com deficiência da UFPA, nas suas variáveis sociodemográficas e acadêmicas, posteriormente os dados relativos aos níveis de resiliência e de estresse e as respectivas correlações com as variáveis de investigação.
A população foi composta por 50 estudantes com deficiência e com transtornos, sendo 28 pertencentes ao sexo masculino (56\%) e 22 ao sexo feminino (44\%). Verificou-se que 20 alunos (40\%) eram estudantes com Baixa Visão ou Cegueira, 18 (36\%) com Mobilidade Reduzida e Cadeirante, cinco (10\%) com Deficiência Auditiva e Surdez e sete (14\%) com Outras $^{2}$. A faixa etária dos estudantes variou entre 18 e 45 anos, com a média de idade dos participantes de 27 anos. Em relação à área de conhecimento, 26 (52\%) estudantes eram das áreas de Filosofia Ciências Humanas, de Sociais Aplicadas e Jurídicas, 14 (28\%) de Exatas e Naturais e 10 (20\%) da Saúde. Dos 50 participantes, 21 estudantes obtiveram níveis abaixo ou igual à média $(\leq 27)$ e foram classificados com baixo nível de estresse. 29 discentes alcançaram níveis acima da média ( $>27$ ) e foram classificados com alto nível de estresse.

Os valores do nível descritivo (p) menores que o nível de significância de 0,05 (5\%) e do Critério Beta $(\beta)$ maior que 3 , indicaram que tanto os índices como suas categorias são dependentes. Além disso, pôde-se observar que a soma dos percentuais de inércia indicou que mais de $70 \%$ da informação foi restituída pela AC. Desta forma todos os pressupostos para utilização da técnica de AC foram satisfeitos. Assim, procedeu-se a correlação dos níveis de estresse e de resiliência com as variáveis sociodemográficos e acadêmicas dos participantes da pesquisa, que resultou em probabilidades moderadamente e fortemente significativas, apresentadas nas Tabelas $1,2,3,4$ e 5 . Os achados dessa pesquisa foram organizados da seguinte forma:

\section{(1) Cruzamento do baixo e alto estresse com os dados sociodemográficos e acadêmicos dos estudantes}

Observa-se na Tabela 1, que o alto estresse dos discentes apresentou associação fortemente significativa com as áreas das Exatas e Naturais $(95,62)^{*}$. Já em relação à deficiência, o alto estresse apresentou associação moderadamente significativa com a Mobilidade Reduzida ou Cadeirante $(59,73)^{* *}$, com a Deficiência Auditiva ou Surdez $(67,89)^{* *}$ e com Outras $(54,96)^{* *}$. O alto estresse também apresentou associação fortemente significativa em alunos com

${ }^{2}$ Outras: três estudantes com dislexia, dois alunos com deficiências múltiplas e dois estudantes com transtorno do espectro autista, totalizando sete participantes. 
Tabela 1

Distribuição do cruzamento do alto e baixo estresse com os dados sociodemográficos e acadêmicos dos estudantes.

\begin{tabular}{|c|c|c|c|}
\hline \multirow{2}{*}{ Variáveis } & \multirow{2}{*}{ Categorias } & \multicolumn{2}{|c|}{ Estresse } \\
\hline & & Alto & Baixo \\
\hline \multirow{4}{*}{ Área de Conhecimento } & $\begin{array}{l}\text { Filosofia e Ciências } \\
\text { Humanas }\end{array}$ & $0,42(32,43)$ & $-0,44(0,00)$ \\
\hline & Saúde & $-1,05(0,00)$ & $1,10(72,67)^{*}$ \\
\hline & Sociais Aplicadas e Jurídicas & $-2,12(0,00)$ & $2,20(97,25)^{*}$ \\
\hline & Exatas e Naturais & $2,02(95,62)^{*}$ & $-2,10(0,00)$ \\
\hline Sexo & $\begin{array}{l}\text { Feminino } \\
\text { Masculino }\end{array}$ & \multicolumn{2}{|c|}{$\mathrm{p}=0,523$} \\
\hline \multirow{4}{*}{ Deficiências e Outras } & $\begin{array}{l}\text { Mobilidade Reduzida/ } \\
\text { Cadeirante }\end{array}$ & $0,84(59,73)^{* *}$ & $-0,87(0,00)$ \\
\hline & Baixa Visão/Cegueira & $-1,74(0,00)$ & $1,81(92,93)^{*}$ \\
\hline & Deficiência Auditiva/Surdez & $0,99(67,89)^{* *}$ & $-1,03(0,00)$ \\
\hline & Outras & $0,75(54,96)^{* *}$ & $-0,79(0,00)$ \\
\hline \multirow{2}{*}{ Faixa Etária } & 18 a 21 & $1,91(94,34)^{*}$ & $-1,98(0,00)$ \\
\hline & 22 a 28 & $0,26(20,60)$ & $-0,27(0,00)$ \\
\hline
\end{tabular}

** Probabilidade moderadamente significativa, pois $50 \% \leq \mathrm{y}$ x $100<70 \%$

* Probabilidade fortemente significativa, pois y x $100 \geq 70 \%$

idade entre 18 a 21 anos $(94,34)^{*}$. Em contraste com esse resultado, o baixo estresse dos estudantes participantes da pesquisa apresentou associação fortemente significativa com as áreas da Saúde $(72,67)^{*}$ e das Sociais Aplicadas e Jurídicas $(97,25)^{*}$, com a Baixa Visão ou Cegueira $(92,93)^{*}$ e com as idades entre 29 a 45 anos $(97,29)^{*}$.

\section{(2) Cruzamento da resiliência geral com os dados sociodemográficos e acadêmicos dos estudantes}

Observa-se na Tabela 2 que alto nível de resiliência dos estudantes apresentou relação fortemente significativa com as áreas da Saúde $(91,75)^{*}$, das Sociais Aplicadas e Jurídicas $(79,80)^{*}$, com o sexo feminino $(96,77)^{*}$, com a Deficiência Auditiva ou Surdez $(99,71)^{*}$ e alunos com idades entre 29 a 45 anos $(99,56)^{*}$. Já o nível moderado de resiliência dos discentes apresentou associação fortemente significativa com a Baixa Visão ou Cegueira $(98,86)^{*}$ e com o sexo masculino $(96,75)^{*}$. No que se refere ao baixo nível de resiliência, os estudantes apresentaram associação fortemente significativa com as áreas da Filosofia Ciências Humanas $(89,40)^{*}$, com o sexo feminino $(78,99)^{*}$, com Outras $(99,97)^{*}$ e com idades entre 18 a 21 anos $(90,59)^{*}$. O baixo nível de resiliência também apresentou associação moderadamente significativa com as áreas das Exatas e Naturais $(51,50)^{* *}$.

\section{(3) Cruzamento dos fatores de resiliência com os tipos de deficiência e outras}

A Tabela 3 apresenta a correlação dos fatores de resiliência com os tipos de deficiências e outras. A Escala de Resiliência (Pesce et al., 2005) possui três fatores: o fator 1 apresenta questões relacionadas com a Competência Pessoal e Aceitação de si e da vida. O fator 2 apresenta questões acerca da Independência e Determinação. $O$ fator 3 apresenta questões que abordam Autoconfiança e Capacidade de adaptação a situações da vida. Os escores obtidos nos fatores 1 , 2 e 3 foram categorizados em Alto, Moderado e Baixo. O Fator 1 apresentou os escores 78,63 a 100 (Alto), 40,52 a 78,63 (Moderado) e 0 a 40,51 (Baixo). No Fator 2 os resultados obtidos foram 93,30 a 100 (Alto), 50,61 a 93,29 (Moderado) e 0 a 50,60 (Baixo). Já os escores do Fator 3 foram classificados em Alto (71,52 a 100), Moderado (33,09 a 71,51) e Baixo (0 a 33,08). 
Tabela 2

Distribuição do cruzamento da resiliência geral com os dados sociodemográficos e acadêmicos dos estudantes.

\begin{tabular}{|c|c|c|c|c|}
\hline \multirow{2}{*}{ Variáveis } & \multirow{2}{*}{ Categorias } & \multicolumn{3}{|c|}{ Resiliência Geral } \\
\hline & & Alto & Moderado & Baixo \\
\hline \multirow{4}{*}{$\begin{array}{l}\text { Área de } \\
\text { Conhecimento }\end{array}$} & $\begin{array}{l}\text { Filosofia e Ciências } \\
\text { Humanas }\end{array}$ & $-1,55(0,00)$ & $0,00(0,00)$ & $1,62(89,40)^{*}$ \\
\hline & Saúde & $1,74(91,75)^{*}$ & $0,00(0,00)$ & $-1,81(0,00)$ \\
\hline & $\begin{array}{l}\text { Sociais Aplicadas e } \\
\text { Jurídicas }\end{array}$ & $1,28(79,80)^{*}$ & $0,00(0,00)$ & $-1,33(0,00)$ \\
\hline & Exatas e Naturais & $-0,67(0,00)$ & $0,00(0,00)$ & $0,70(51,50)^{* *}$ \\
\hline \multirow{2}{*}{ Sexo } & Feminino & $2,14(96,77)^{*}$ & $-2,41(0,00)$ & $1,25(78,99)^{*}$ \\
\hline & Masculino & $-1,90(0,00)$ & $2,14(96,75)^{*}$ & $-1,11(0,00)$ \\
\hline \multirow{4}{*}{ Deficiência e Outras } & & $0,30(23,26)$ & $-0,67(0,00)$ & $0,65(48,71)$ \\
\hline & Baixa Visão/Cegueira & $-1,05(0,00)$ & $2,53(98,86)^{*}$ & $-2,56(0,00)$ \\
\hline & $\begin{array}{l}\text { Deficiência Auditiva/ } \\
\text { Surdez }\end{array}$ & $2,98(99,71)^{*}$ & $-1,90(0,00)$ & $-0,37(0,00)$ \\
\hline & Outras & $-1,22(0,00)$ & $-1,60(0,00)$ & $3,58(99,97)^{*}$ \\
\hline \multirow{3}{*}{ Faixa Etária } & 18 a 21 & $-1,02(0,00)$ & $-0,43(0,00)$ & $1,67(90,59)^{*}$ \\
\hline & 22 a 28 & $-1,35(0,00)$ & $0,55(42,09)$ & $0,61(45,71)$ \\
\hline & 29 a 45 & $2,85(99,56)^{*}$ & $-0,39(0,00)$ & $-2,40(0,00)$ \\
\hline
\end{tabular}

** Probabilidade moderadamente significativa, pois $50 \% \leq \mathrm{y}$ x $100<70 \%$

* Probabilidade fortemente significativa, pois y x $100 \geq 70 \%$

Tabela 3

Distribuição do cruzamento dos fatores de resiliência (fatores 1, 2 e 3) com os tipos de deficiências e outras.

\begin{tabular}{lccc}
\hline \multirow{2}{*}{ Categorias } & \multicolumn{1}{c}{ Fator 1} & Baixo \\
\cline { 2 - 4 } & Alto & Moderado & $-0,31(0,00)$ \\
Baixa Visão/Cegueira & $-0,31(0,00)$ & $0,42(32,43)$ & $-1,64(0,00)$ \\
Deficiência Auditiva/Surdez & $1,10(72,67)^{*}$ & $0,37(29,02)$ & $-0,37(0,00)$ \\
Outras & $-0,37(0,00)$ & $0,50(38,02)$ & $3,58(99,97)^{*}$ \\
\hline \multirow{2}{*}{ Categorias } & $-1,05(0,00)$ & $-1,72(0,00)$ & Baixo \\
\hline & & Fator 2 & $-0,31(0,00)$ \\
Baixa Visão/Cegueira & Alto & $0,42(32,43)$ & $-2,56(0,00)$ \\
Deficiência Auditiva/Surdez & $-0,31(0,00)$ & $1,61(89,31)^{*}$ & $-0,37(0,00)$ \\
Outras & $0,18(14,49)$ & $-1,98(0,00)$ & $5,12(100,00)^{*}$ \\
\hline Categorias & $3,29(99,90)^{*}$ & $-1,72(0,00)$ & Baixo \\
\hline & $-2,59(0,00)$ & Fator 3 & $1,62(89,40)^{*}$ \\
Baixa Visão/Cegueira & & Moderado & $-2,56(0,00)$ \\
Deficiência Auditiva/Surdez & Alto & $-0,89(0,00)$ & $1,46(85,59)^{*}$ \\
Outras & $-0,31(0,00)$ & $0,99(67,89)^{* *}$ & $0,49(37,85)$ \\
\hline
\end{tabular}

** Probabilidade moderadamente significativa, pois $50 \% \leq \mathrm{y}$ x $100<70 \%$

* Probabilidade fortemente significativa, pois y x $100 \geq 70 \%$ 
A Tabela 3 mostra o cruzamento dos tipos de deficiência e outras com os Fatores da Escala de Resiliência. Os alunos com Baixa Visão ou Cegueira apresentaram associação fortemente significativa com os níveis alto de resiliência no que se referem ao fator $1(72,67)^{*}$ e ao fator $3(72,67)^{*}$. Em relação ao fator 2, esses estudantes apresentaram uma relação fortemente significativa com os níveis de resiliência moderado $(89,31)^{*}$. Os estudantes com Outras apresentaram associação fortemente significativa com níveis baixos de resiliência no fator $1(99,97)^{*}$ e no fator $2(100,00)^{*}$. Já em relação aos alunos com Deficiência Auditiva ou Surdez, eles apresentaram relação fortemente significativa com níveis de resiliência alta no fator $2(99,90)^{*}$ e níveis de resiliência baixo no fator $3(85,59)^{*}$. Por fim, os alunos com Mobilidade Reduzida ou Cadeirante apresentaram associação fortemente significativa com níveis de resiliência baixo no fator $3(89,40)^{*}$.

\section{(4) Cruzamento dos Fatores 1, $2 \mathrm{e}$ 3 com os dados sociodemográficos e acadêmicos dos estudantes}

Observa-se na Tabela 4 que o alto nível de resiliência do fator 1 apresentou associação fortemente significativa com as áreas da Saúde $(87,87)^{*}$ e das Exatas e Naturais $(83,75)^{*}$, com o sexo masculino $(95,17)^{*}$ e com as idades entre 29 a 45 anos $(99,89)^{*}$. Já o nível moderado do fator 1 mostrou relação fortemente significativa com as áreas das Sociais Aplicadas e Jurídicas $(90,05)^{*}$ e com as faixas etárias entre 81 a $21(71,56)^{*}$. Apresentou também associação moderadamente significativa com as idades entre 22 a 28 anos $(57,92)^{*}$. O nível baixo do fator 1 apresentou associação fortemente significativa com as áreas de Filosofia Ciências Humanas $(99,88)^{*}$ e com o sexo feminino $(78,99)^{*}$.

Verifica-se na Tabela 4 que o alto nível de resiliência do fator 2 apresentou associação fortemente significativa com as áreas das Exatas e Naturais $(83,75)^{*}$ e com o sexo feminino $(98,21)^{*}$. Em relação à faixa etária dos estudantes, o alto nível do fator 2 apresentou associação moderadamente significativa com as idades entre 29 a 45 anos $(68,09)^{* *}$. Já o nível moderado do fator 2 mostrou relação fortemente significativa com as áreas da Saúde $(99,84)^{*}$ e com o sexo masculino $(98,00)^{*}$. O baixo nível do fator 2 apresentou associação fortemente significativa com as áreas de Filosofia Ciências Humanas $(99,88)^{*}$, com o sexo feminino $(86,56)^{*}$ e com as idades entre 18 a 21 anos $(90,59)^{*}$.

Observa-se, ainda, na Tabela 4 que o alto nível de resiliência do fator 3 apresentou associação moderadamente significativa com as áreas da Saúde $(56,14)^{* *}$ e fortemente significativa com as áreas das Sociais Aplicadas e Jurídicas $(88,10)^{*}$ e com a faixa etária entre 29 a 45 anos $(96,67)^{*}$. Já o nível moderado do fator 3 mostrou relação moderadamente significativa com as áreas da Saúde $(51,71)^{* *}$ e com as Sociais Aplicadas e Jurídicas $(58,99)^{* *}$ e associação fortemente significativa com o sexo masculino $(93,49)^{*}$. Verifica-se na Tabela 4 que o nível moderado do fator 3 apresentou associação fortemente significativa com as idades entre 22 a 28 anos $(82,26)^{*}$. O baixo nível do fator 3 apresentou associação fortemente significativa com as áreas de Exatas e Naturais $(99,60)^{*}$, com o sexo masculino $(95,17)^{*}$ e com as idades entre 18 a 21 anos $(90,59)^{*}$.

\section{(5) Cruzamento do Estresse com a Resiliência e seus Fatores}

Observa-se na Tabela 5 que o alto estresse dos discentes participantes da pesquisa apresentou associação fortemente significativa com a baixa resiliência $(84,12)^{*}$. No que se refere ao fator 1 , o alto estresse apresentou relação fortemente significativa com o baixo nível de Competência pessoal e Aceitação de si e da vida $(84,12)^{*}$. No que tange ao fator 2 , $\mathrm{o}$ alto estresse mostrou associação fortemente significativa com o nível moderado de Independência e Determinação $(89,26)^{*}$. Já no que diz respeito ao fator 3, o alto estresse apresentou relação fortemente significativa com o baixo $(84,12) *$ e o alto $(84,12)^{*}$ níveis de Autoconfiança e Capacidade de Adaptação a situações da vida. Em contraste com esse resultado, o baixo estresse dos alunos com deficiência apresentou associação fortemente significativa com a alta resiliência $(97,29)^{*}$. No que se refere aos fatores $1 \mathrm{e}$ 2, o baixo estresse apresentou relação fortemente significativa com o alto nível de Competência pessoal e Aceitação de si e da vida $(93,81)^{*}$ e de Independência e Determinação $(96,12)^{*}$. No que tange ao fator 3, o baixo estresse apresentou associação fortemente significativa com o nível moderado de Autoconfiança e Capacidade de adaptação a situações da vida $(95,37)^{*}$. 
Tabela 4

Distribuição do cruzamento dos níveis de resiliência (alto, moderado e baixo) dos fatores 1, 2 e 3 com os dados sociodemográficos e acadêmicos dos estudantes.

\begin{tabular}{|c|c|c|c|c|}
\hline \multirow{2}{*}{ Variáveis } & \multirow{2}{*}{ Categorias } & \multicolumn{3}{|c|}{ Fator 1} \\
\hline & & Alto & Moderado & Baixo \\
\hline \multirow[t]{4}{*}{$\begin{array}{l}\text { Área de } \\
\text { Conhecimento }\end{array}$} & & $-2,54(0,00)$ & $-0,47(0,00)$ & $3,23(99,88)^{*}$ \\
\hline & Saúde & $1,55(87,87)^{*}$ & $-0,35(0,00)$ & $-1,03(0,00)$ \\
\hline & $\begin{array}{l}\text { Sociais Aplicadas e } \\
\text { Jurídicas }\end{array}$ & $0,23(18,26)$ & $1,65(90,05)^{*}$ & $-2,66(0,00)$ \\
\hline & Exatas e Naturais & $1,40(83,75)^{*}$ & $-0,42(0,00)$ & $-0,79(0,00)$ \\
\hline \multirow[t]{2}{*}{ Sexo } & Feminino & $-2,23(0,00)$ & $0,66(49,22)$ & $1,25(78,99) *$ \\
\hline & Masculino & $1,98(95,17)^{*}$ & $-0,59(0,00)$ & $-1,11(0,00)$ \\
\hline \multirow[t]{3}{*}{ Faixa Etária } & 18 a 21 & $-0,79(0,00)$ & $1,07(71,56)^{*}$ & $-0,79(0,00)$ \\
\hline & 22 a 28 & $-1,79(0,00)$ & $0,81(57,92)^{* *}$ & $0,61(45,71)$ \\
\hline & 29 a 45 & $3,26(99,89)$ & $-2,12(0,00)$ & $-0,14(0,00)$ \\
\hline \multirow{2}{*}{ Variáveis } & \multirow{2}{*}{ Categorias } & \multicolumn{3}{|c|}{ Fator 2} \\
\hline & & Alto & Moderado & Baixo \\
\hline \multirow[t]{4}{*}{$\begin{array}{l}\text { Área de } \\
\text { Conhecimento }\end{array}$} & & $-0,62(0,00)$ & $-1,78(0,00)$ & $3,23(99,88)^{*}$ \\
\hline & Saúde & $-1,03(0,00)$ & $3,16(99,84)^{*}$ & $-3,61(0,00)$ \\
\hline & $\begin{array}{l}\text { Sociais Aplicadas e } \\
\text { Jurídicas }\end{array}$ & $0,23(18,26)$ & $-0,31(0,00)$ & $0,23(18,26)$ \\
\hline & Exatas e Naturais & $1,40(83,75)^{*}$ & $-0,42(0,00)$ & $-0,79(0,00)$ \\
\hline \multirow[t]{2}{*}{ Sexo } & Feminino & $2,37(98,21)^{*}$ & $-2,63(0,00)$ & $1,50(86,56)^{*}$ \\
\hline & Masculino & $-2,10(0,00)$ & $2,33(98,00)^{*}$ & $-1,33(0,00)$ \\
\hline \multirow[t]{3}{*}{ Faixa Etária } & 18 a 21 & $-2,02(0,00)$ & $0,23(18,51)$ & $1,67(90,59)^{*}$ \\
\hline & 22 a 28 & $0,61(45,71)$ & $-0,28(0,00)$ & $-0,19(0,00)$ \\
\hline & 29 a 45 & $1,00(68,09)^{* *}$ & $0,18(14,65)$ & $-1,27(0,00)$ \\
\hline \multirow{2}{*}{ Variáveis } & \multirow{2}{*}{ Categorias } & \multicolumn{3}{|c|}{ Fator 3} \\
\hline & & Alto & Moderado & Baixo \\
\hline \multirow[t]{4}{*}{$\begin{array}{l}\text { Área de } \\
\text { Conhecimento }\end{array}$} & & $-1,27(0,00)$ & $0,42(32,43)$ & $0,65(48,71)$ \\
\hline & Saúde & $0,77(56,14)^{*}$ & $0,70(51,71)^{*}$ & $-1,81(0,00)$ \\
\hline & $\begin{array}{l}\text { Sociais Aplicadas e } \\
\text { Jurídicas }\end{array}$ & $1,56(88,10)^{*}$ & $0,82(58,99)^{*}$ & $-2,77(0,00)$ \\
\hline & Exatas e Naturais & $-0,39(0,00)$ & $-1,69(0,00)$ & $2,88(99,60)^{*}$ \\
\hline \multirow[t]{2}{*}{ Sexo } & Feminino & $-0,49(0,00)$ & $1,84(93,49)^{*}$ & $-2,23(0,00)$ \\
\hline & Masculino & $0,43(33,43)$ & $-1,64(0,00)$ & $1,98(95,17)^{*}$ \\
\hline \multirow[t]{3}{*}{ Faixa Etária } & 18 a 21 & $-0,79(0,00)$ & $-0,60(0,00)$ & $1,67(90,59)^{*}$ \\
\hline & 22 a 28 & $-0,99(0,00)$ & $1,35(82,26)^{*}$ & $-0,99(0,00)$ \\
\hline & 29 a 45 & $2,13(96,67)^{*}$ & $-1,35(0,00)$ & $-0,14(0,00)$ \\
\hline
\end{tabular}

** Probabilidade moderadamente significativa, pois $50 \% \leq$ y x $100<70 \%$

* Probabilidade fortemente significativa, pois y x $100 \geq 70 \%$ 
Tabela 5

Distribuição do cruzamento do baixo e alto estresse com a resiliência e com os fatores 1, 2 e 3 .

\begin{tabular}{|c|c|c|c|}
\hline \multirow{2}{*}{ Variáveis } & \multirow{2}{*}{ Categorias } & \multicolumn{2}{|c|}{ Estresse } \\
\hline & & Alto & Baixo \\
\hline \multirow{3}{*}{ Resiliência } & Baixa & $1,41(84,12)^{*}$ & $-1,47(0,00)$ \\
\hline & Moderada & $0,55(42,09)$ & $-0,58(0,00)$ \\
\hline & Alta & $-2,12(0,00)$ & $2,21(97,29) *$ \\
\hline \multirow{3}{*}{ Fator 1} & Baixo & $1,41(84,12)^{*}$ & $-1,47(0,00)$ \\
\hline & Moderado & $0,26(20,60)$ & $-0,27(0,00)$ \\
\hline & Alto & $-1,79(0,00)$ & $1,87(93,81)^{*}$ \\
\hline \multirow{3}{*}{ Fator 2} & Baixo & $-0,38(0,00)$ & $0,40(31,08)$ \\
\hline & Moderado & $1,61^{*}(89,26)^{*}$ & $-1,68(0,00)$ \\
\hline & Alto & $-1,99(0,00)$ & $2,07(96,12)^{*}$ \\
\hline \multirow{3}{*}{ Fator 3} & Baixo & $1,41(84,12)^{*}$ & $-1,47(0,00)$ \\
\hline & Moderado & $-1,91(0,00)$ & $1,99(95,37)^{*}$ \\
\hline & Alto & $1,41(84,12)^{*}$ & $-1,47(0,00)$ \\
\hline
\end{tabular}

* Probabilidade fortemente significativa, pois y x $100 \geq 70 \%$

\section{Discussão}

Os resultados dessa pesquisa mostraram que a maioria dos estudantes com Deficiência Auditiva ou Surdez, Mobilidade Reduzida ou Cadeirante e Outras que estudam na UFPA apresentou níveis elevados e moderados de estresse. Esses alunos são dos cursos de Exatas e Naturas e com idades entres 18 a 21 anos. Tal resultado pode estar relacionado à imaturidade dos alunos ainda muito jovens, com a maneira como as aulas e as avaliações são administradas nos cursos escolhidos, com a falta de preparo da instituição em relação aos recursos tecnológicos e metodológicos e com a inexperiência dos professores para lidar com essas deficiências e transtornos. A esse respeito, Benevides (2011) revela sua preocupação com a responsabilidade dos professores que ministram disciplinas para alunos com deficiência. Para o autor, a metodologia e a avaliação da aprendizagem, sem o uso de instrumentos avaliativos variados acaba desconsiderando as particularidades do aluno com deficiência.

Apesar de encontrar poucos estudos na literatura que tratam dessa temática no ensino superior, esse resultado corrobora os achados de Atabey, Karanci, Dirik e Aydemir (2011), Heiman (2006) e Hong (2015) que investigaram os níveis de estresse em alunos com deficiência no ensino superior e concluíram que os mesmos apresentaram níveis altos de estresse. Para os autores, oferecer programas para promover a inclusão e mudanças nas barreiras dos ambientes acadêmico e físico dos campi universitários pode revelar-se eficaz na diminuição dos níveis de estresse nesse público.

Estudos que tratam do estresse em alunos sem deficiência no contexto acadêmico também corroboram o resultado encontrado nesta pesquisa. Os estudos de Lameu, Salazar, \& Souza, 2016; Milsted, Amorim, \& Santos, 2014; Mondardo, \& Pedon, 2005; Monteiro, Freitas, \& Ribeiro, 2007; Santos et al, 2012; Thomaz, Rocha, \& Neto, 2011 analisaram os níveis de estresse em acadêmicos sem deficiência e consideraram suas atividades na universidade como fontes geradoras de estresse. Os autores sugerem a adoção de medidas de prevenção e apoio psicológico que detectem situações que favorecem o estresse, assim como, ajustes na estrutura pedagógica.

De fato, os dados ressaltam a importância da variável idade na medida em que revela que alunos com Baixa Visão ou Cegueira, dos cursos de Saúde, Sociais e Aplicadas e Jurídicas, com idades entre 29 a 45 anos apresentaram níveis baixos de estresse. É possível que este resultado esteja associado à 
maturidade dos alunos que quanto mais velhos, estão mais preparados para lidar com os desafios. A esse respeito, os dados da presente pesquisa corroboram os estudos de Cecconello e Koller (2000) que afirmam que pessoas mais velhas têm maior capacidade de iniciativa quando comparadas as mais jovens, utilizando-se da empatia e da capacidade de iniciativa como características resilientes.

Em se tratando da resiliência, os resultados desta pesquisa mostraram que a maioria dos alunos do sexo feminino, com Deficiência Auditiva ou Surdez, entre as idades de 29 a 45 anos e dos cursos de Saúde e Sociais Aplicadas e Jurídicas apresentaram níveis elevados de resiliência. Este resultado pode estar associado às características que a pessoa resiliente apresenta como adaptabilidade, baixa suscetibilidade, enfrentamento, resistência à destruição, condutas vitais positivas, temperamento especial e habilidades cognitivas. Todas essas características seriam desenvolvidas durante situações vitais adversas, e estressantes permitindo aos indivíduos atravessá-las e superá-las (Melillo, Estamatti, $\&$ Cuestas, 2005).

Em oposição a esse resultado, os baixos níveis de resiliência foram correlacionados com os alunos dos cursos de Filosofia Ciências Humanas e Exatas e Naturais, do sexo feminino, com idades entre 18 a 21 anos e com Outras (Dislexia, Autismo e Deficiências Múltiplas). A baixa resiliência pode estar associada à imaturidade dos alunos, já que tinham idades entre 18 a 21 anos. Para Garmezy e Masten (1994), estas pessoas estão vulneráveis a potencializar os efeitos de um evento de risco (estressor) deixando o indivíduo suscetível a consequências negativas para o desenvolvimento psicológico (Zimmerman, \& Arunkumar, 1994). Para Antoni e Koller (2000), a prematuridade, dificuldade de acesso a saúde e educação, evasão escolar, problemas de aprendizagem, déficit de atenção, isolamento e falta de responsabilidade podem interferir no comportamento das pessoas, contribuindo para os baixos níveis de resiliência. Pode-se também concluir que o baixo nível de resiliência possa estar associado às dificuldades de relacionamento, a discriminação e ao preconceito, assim como as barreiras físicas e a falta de recursos (Lima, 2013).

Sobre o cruzamento dos fatores da resiliência com as variáveis sexo, área de conhecimento, idade, deficiência e outras, a presente pesquisa mostrou que a maioria dos alunos com Baixa Visão ou Cegueira e Deficiência Auditiva ou Surdez, com idades entre 29 a 45 anos, dos cursos de Saúde, Exatas e Naturais e Sociais Aplicadas e Jurídicas e do sexo masculino e feminino apresentaram níveis elevados de resiliência nas três categorias do fator 1,2 e 3 , o que corresponde aos altos níveis de resiliência das dimensões de competência pessoal e aceitação de si, determinação e independência e autoconfiança e capacidade de adaptação as situações. Este resultado mostra que o desenvolvimento de capacidades de resiliência nas pessoas passa pela mobilização e ativação das suas capacidades de ser, estar, ter, poder e querer, ou seja, pela sua capacidade de autorregulação e autoestima (Tavares, 2002).

Por outro lado, os alunos com Deficiência Auditiva ou Surdez, Mobilidade Reduzida ou Cadeirante e Outras, dos cursos de Exatas e Naturais e Filosofia Ciências Humanas, com idades entre 18 a 21 anos e dos sexos masculino e feminino apresentaram níveis baixos de resiliência do fator 1,2 e 3, o que indica provavelmente que estes alunos não estão demonstrando características relacionadas à capacidade de resolver sozinho situações difíceis, lidar com várias situações ao mesmo tempo, aceitar as adversidades e aceitar situações que não pode fazer nada para mudar (Pesce et al., 2005).

Já a maioria dos estudantes com Baixa Visão ou Cegueira, dos cursos de Saúde e Sociais Aplicadas e Jurídicas, com idades entre 18 a 28 anos e de ambos os sexos apresentaram níveis moderados de resiliência dos fatores 1, 2 e 3, o que provavelmente demonstra que estes discentes têm características moderadas de autoconfiança e capacidade de adaptação a situações. $O$ resultado indica que esses alunos precisam acreditar que podem resolver seus problemas e que tais problemas dependem mais deles do que dos outros para serem resolvidos.

No presente estudo, a correlação dos níveis de estresse com os níveis de resiliência e com os fatores 1,2 e 3 demonstraram que alunos que apresentaram níveis altos de estresse, são alunos com níveis moderados e baixos de resiliência. Com este dado, percebeu-se que esses alunos além de estressados, apresentaram níveis moderados e baixos de competência pessoal e aceitação de si, de independência e determinação e de autoconfiança, assim como de capacidade de adaptação a situações da vida (Pesce et al., 2005). 
Este resultado sugere que os participantes desse estudo não estão conseguindo superar as adversidades do contexto universitário. As adversidades, segundo Sapienza e Pedromônico (2005), não costumam acontecer isoladamente, já que fazem parte do contexto social, envolvendo fatores políticos, socioeconômicos, ambientais, culturais e familiares. Assim, os fatores de risco, quando em associação interativa, constituem-se em mecanismos de risco, aumentando a probabilidade ou desencadeando um desfecho negativo no desenvolvimento do indivíduo. Quando muitas situações de risco se associam, elas dificultam o desenvolvimento, a aquisição de habilidades e o desempenho de papéis sociais.

Os dados desta pesquisa vão ao encontro dos resultados obtidos das pesquisas (Heiman, 2006; Hong, Haefner, \& Slekar, 2011; Getzel, \& Thoma, 2008; Mamiseishvili, \& Koch, 2011; Reed, Kennett, \& Emond, 2015) que encontraram níveis de resiliência baixa em estudantes com deficiência no ensino superior. Os autores constataram que o suporte do docente ajudaria o estudante a desenvolver habilidades de autodeterminação. Para os pesquisadores, o aluno com deficiência precisa ser um defensor de seus interesses, isto é, saber resolver problemas, ser autocrítico em relação a si próprio, acreditar nos seus objetivos, saber gerenciar seu tempo e atividades acadêmicas e ser consciente de suas competências e dificuldades escolares. Nesta direção, percebeu-se a importância que a instituição de ensino tem na transmissão de valores e no desenvolvimento de comportamentos resilientes para o enfrentamento e resolução de problemas.

A adaptação do estudante ao ensino superior pode ser reforçada se o clima institucional for promotor da aprendizagem e do desenvolvimento (Soares, Almeida \& Guisande, 2011). As investigações na área têm demonstrado que professores que recorrem a práticas e estratégias de ensino, a métodos de avaliação focados nas competências e a mecanismos de feedback positivos contribuem para a adaptação acadêmica do estudante (Soares et al., 2011).

Neste sentido, entende-se que as universidades devem a partir de programas e serviços de apoio, com psicológicos, pedagógicos e professores, promover o envolvimento do estudante nas atividades acadêmicas, pessoais, curriculares e/ou extracurriculares, estimular o desenvolvimento pessoal, psi- cossocial e a saúde mental do mesmo, maximizar as suas competências cognitivas, desenvolver métodos de estudo e de ensino e aprendizagem e estratégias focadas na resolução dos seus problemas e intervir terapeuticamente em psicopatologias impeditivas de uma adaptação saudável do aluno neste nível de ensino (Almeida, \& Cruz, 2010; Cabral, 2011; Soares et al., 2011).

\section{Considerações finais}

O presente estudo teve como objetivo central analisar a correlação entre os níveis de estresse com os níveis de resiliência em alunos com deficiência da UFPA. Os achados desta pesquisa contribuíram para o avanço da discussão na temática, assim como colaborou para a ampliação da compreensão acerca destas variáveis neste nível de ensino, tão relevantes para o entendimento do desenvolvimento humano e do enfrentamento frente às adversidades.

Como limitação do estudo, destaca-se que a literatura que trata da correlação do estresse e de características resilientes de estudantes com deficiência no ensino superior ainda é escasso, dificultando a comparação com outros estudos empíricos nacionais e internacionais.

Concluiu-se que uma instituição de ensino resiliente intervém de maneira positiva no contexto acadêmico quando favorece um processo de mudança que aumente e resgate autoestima dos alunos estimulando-os a resistir ao estresse e as tensões a fim de que superem as incertezas e adversidades impostas pelo cotidiano acadêmico.

Como a pesquisa se concentrou somente no campus do Guamá, sugere-se para futuras pesquisas, a inclusão de outros campi que compõe a Universidade Federal do Pará para melhor conhecer a realidade desses alunos o que permitirá o planejamento de intervenções voltadas para diminuição de problemas e a promoção da resiliência. Por fim, espera-se que este estudo possa contribuir com a construção de políticas institucionais que favoreça a inclusão de fato desse público na Universidade Federal do Pará e em outras instituições de ensino superior. 
Pereira, R. R., Silva, S. S. C., Faciola, R. A., Pontes, F. A. R., Ramos, M. F. H., Soares, E. M. L. (2019). Estresse e Características Resilientes em Alunos com Deficiência.

\section{Referências}

Almeida, L. S., \& Cruz, J. F. A. (2010). Transição e adaptação acadêmica: Reflexões em torno dos alunos do $1^{\circ}$ ano da Universidade do Minho. In: Comunicação apresentada no Congresso Ibérico Ensino Superior em Mudança: Tensões e Possibilidades. Braga: Universidade do Minho. Recuperado de http://hdl.handle. net/1822/11543

Antoni, C., \& Koller, S. H. (2000). Vulnerabilidade e resiliência familiar: Um estudo com adolescentes que sofreram maus tratos intrafamiliares. Psico, 31(1), 39-66.

Atabey, M. K., Karanci, A. N., Dirik, G., \& Aydemir, D. (2011). Psychological wellbeing of turkish university students with physical impairments: An evaluation within the stress-vulnerability paradigm. International Journal of Psychology, 46(2), 106-118. https:// doi.org/10.1080/00207594.2010.513413

Barroco, S. M. S. (2003). Educação inclusiva e prática social excludente: Reflexões necessárias. In: Anais da Jornada internacional de Psicologia e Encontro Paranaense de Psicologia Social. Umuarama, PR: Abrapso.

Benevides, P. S. (2011). As retóricas contemporâneas e a significação de educação inclusiva. Psicologia \& Sociedade, 23(2), 248-253. https://doi.org/10.1590/S0102-71822011000200005

Brasil. (2008). Política nacional de educação especial na perspectiva da educação inclusiva. Brasília, DF: MEC/ SECADI. Recuperado de http://portal.mec.gov.br/arquivos/pdf/politicaeducespecial.pdf.

Baptista, M. N., \& Campos, L. F. L. (2000). Avaliação longitudinal de sintomas de depressão e estresse em estudantes de psicologia. Boletim de Psicologia, 50(113), 37-58.

Cabral, J. (2011). Vinculação, desenvolvimento psicossocial e adaptação à universidade: Dinâmicas cognitivas-emocionais (Tese de Doutorado). Universidade do Porto, Porto, Portugal.

Cecconello, A. M., \& Koller, S. H. (2000). Competência social e empatia: Um estudo sobre resistência com crianças em situação de pobreza. Estudos de Psicologia, 5(1), 71-93. https://doi.org/10.1590/S1413294X2000000100005

Cohen, S., Karmack, T., \& Mermelsteinm, R. (1983). A global measure of perceived stress. Journal of Health and Social Behavior, 24(4), 385-96. https://doi.org/10.2307/2136404

Fávero, L., Belfiori, P., Silva, F., \& Chan, B. (2009). Análise dos dados: Modelagem multivariada para tomada de decisões. Rio de Janeiro, RJ: Elsever.

Fernandes, E., \& Almeida, L. (2007). Estudantes com deficiência na Universidade: Questões em torno da sua adaptação e sucesso acadêmico. Revista de Educação Especial e Reabilitação, 14, 7-14.

Garmezy, N., \& Masten, A. S. (1994). Chronic adversities. In: M. Rutter, E. Taylor, \& L. Hersov (Eds.), Child and adolescent psychiatry (pp. 191-208, 4th ed.). Oxford: Blackwell Scientific Publications.

Getzel, E. E., \& Thoma, C. A. (2008). Experiences of college students with disabilities and the importance of self-determination in higher. Career Development for Exceptional Individuals, 31(2), 77-84. https://doi. org/10.1177/0885728808317658

Heiman, T. (2006). Social support networks, stress, sense of coherence and academic success of university students with learning disabilities. Social Psychology of Education, 9(4), 461-478. https://doi.org/10.1007/ s11218-006-9007-6

Hong, B., Haefner, L., \& Slekar, T. (2011). Faculty attitudes and knowledge toward promoting self-determination and self-directed learning for college students with and without disabilities. International Journal of Teaching and Learning in Higher Education, 23(2), 175-185.

Hong, B. S. S. (2015). Qualitative analysis of the barriers college students with disabilities experience in higher education. Journal of College Student Development, 56(3), 209-226. https://doi.org/10.1353/csd.2015.0032

Lameu, J. N., Salazar, T. L., \& Souza, W. F. (2016). Prevalência de sintomas de stress entre graduandos de uma universidade pública. Psicologia da Educação, (42), 13-22. https://doi.org/10.5935/2175-3520.20150021

Lima, U. T. S. (2013). Inclusão do aluno com deficiência no ensino superior em saúde nas universidades públicas do estado de Alagoas: Atores, realidade e práticas (Dissertação de Mestrado). Faculdade de Medicina, Universidade Federal de Alagoas, Maceió, AL, Brasil. 
Lipp, M. E. N. (Org.). (2004). Stress no Brasil: Pesquisas avançadas. Campinas, SP: Papirus.

Luft, C. B., Sanches, S. O., Mazo, G. Z., \& Andrade, A. (2007). Versão brasileira da escala de estresse percebido: Tradução e validação para idosos. Revista de Saúde Pública, 41(4), 606-615. https://doi.org/10.1590/S003489102007000400015

Mamiseishvili, K., \& Koch, C. L. (2011). First-to-second-year persistence of students with disabilities in postsecondary institutions in the United States. Rehabilitation Counseling Bulletin, 54(2), 93-105. https://doi. org $/ 10.1177 / 0034355210382580$

Melillo, A., Estamatti, M., \& Cuestas, A. (2005). Alguns fundamentos psicológicos do conceito de resiliência. In: A. Melillo, \& E. N. S. Ojeda (Orgs.), Resiliência: Descobrindo as próprias fortalezas (pp. 59-72). Porto Alegre, RS: Artmed.

Milsted, J. G., Amorim, C., \& Santos, M. (2009). Nível de estresse em alunos de psicologia do período noturno. In: Anais do IX Congresso Nacional de Educação - Educere, III Encontro Sul Brasileiro de Psicopedagogia. Curitiba, PR: PUC-PR. Recuperado de http://www.isad.br/eventos/educere/educere2009/anais/pdf/3289_1469.pdf

Mondardo, A. H., \& Pedon, E. A. (2005). Estresse e desempenho acadêmico em estudantes universitários. Revista de Ciências Humanas, 6(6), 159-180.

Monteiro, C. F. S., Freitas, J. F. M., \& Ribeiro, A. A. P. (2007). Estresse no cotidiano dos alunos de enfermagem da UFPI. Escola Anna Nery, 11(1), 66-72. https:// doi.org/10.1590/S1414-81452007000100009

Mota-Cardoso, R., Araújo, A., Ramos, R., Gonçalves, G., \& Ramos, M. (2002). O stress nos professores portugueses: Estudo IPSSO 2000. Porto: Porto.

Pesce, R., Assis, S. G., Avanci, J., Malaquias, J., \& Oliveira, R. V. C. (2005). Adaptação transcultural, confiabilidade e validade da escala de resiliência. Cadernos de Saúde Pública, 21(2), 436-448. https://doi.org/10.1590/S0102$311 X 2005000200010$

Reed, M. J., Kennett, D. J., \& Emond, M. (2015). The influence of reasons for attending university on university experience: A comparison between students with and without disabilities. Active Learning in Higher Education, 16(3), 1-12. https://doi.org/10.1177/1469787415589626

Sampaio, S. (2005). A psicopedagogia como promovedora de resiliência. Recuperado de http://www.psicopedagogia.com.br/artigos/artigo.asp?entrID=725

Sanchez, P. A. (2005). A educação inclusiva: Um meio de construir escolas para todos no século XXI. Revista Inclusão, 1(1), 7-18.

Santos, J. A. F., Lucena, N. M. G., Rocha, T. V., Aragão, P. O. R., Gatto-Cardia, M. C., Carvalo, A, G. C. et al. (2012). Estresse em acadêmicos do curso de fisioterapia. Revista Brasileira de Ciências da Saúde, 16(supl. 2), 89-94. https://doi.org/10.4034/RBCS.2012.16.s2.12

Sapienza, G., \& Pedromônico, M. R M. (2005). Risco, proteção e resiliência no desenvolvimento da criança e do adolescente. Psicologia em Estudo, 10(2), 209-216.

Silva, N., \& Motta, C. D. V. B. (2009). A criatividade como fator de resiliência na ação docente do professor de ensino superior. Revista UFG, 7(2), 43-46. https://doi.org/10.5216/revufg.v7i2.48869

Soares, A. P., Almeida, L. S., \& Guisande, M. A. (2011). Ambiente académico y adaptación a la universidad: Um estúdio com estudiantes de $1^{\circ}$ año de la Universidad do Minho. Revista Iberoamericana de Psicología y Salud, 2(1), 99-121.

Tavares, J. (2002) A resiliência na sociedade emergente. In: J. Tavares (Org.), Resiliência e educação (pp. 43-76). São Paulo, SP: Cortez.

Thomaz, P. E., Rocha, L. B., \& Neto, V. M. (2011). Estresse em estudantes de engenharia. Momento, 20(1), 73-86.

Vianna, A. M. (2008). Traços de personalidade e fatores de resiliência relacionados ao desenvolvimento de transtorno de estresse pós-traumático subclínico em policiais militares (Dissertação de Mestrado). Faculdade de Ciências Médicas, Santa Casa de São Paulo, São Paulo, SP, Brasil.

Wagnild, G. M., \& Young, H. M. (1993). Development and psychometric evaluation of resilience scale. Journal of Nursing Measurement, 1(2), 165-178. 
Zimmerman, M. A., \& Arunkumar, R. (1994). Resiliency research: Implications for schools and policy. Social Policy Report: Society for Research in Child Development, 8(4), 1-18.

\section{Simone Souza da Costa Silva}

Professora do Programa de Pós-graduação em Teoria e Pesquisa do Comportamento (PPGTPC) da Universidade Federal do Pará, Belém - PA, Brasil.

E-mail: symon.ufpa@gmail.com

(iD) https:// orcid.org/0000-0003-0795-2998

\section{Rosamaria Reo Pereira}

Professora da Faculdade de Letras Estrangeiras Modernas (FALEM) da Universidade Federal do Pará, Belém PA, Brasil.

E-mail: rosareoufpa@gmail.com

(iD) https:// orcid.org/0000-0002-6134-2245

\section{Rosana Assef Faciola}

Professora da Faculdade de Letras Estrangeiras Modernas (FALEM) da Universidade Federal do Pará, Belém PA, Brasil.

E-mail: rosanassef@gmail.com

(iD) https://orcid.org/0000-0002-0987-4527

Fernando Augusto Ramos Pontes

Professor do Programa de Pós-graduação em Teoria e Pesquisa do Comportamento (PPGTPC) da Universidade Federal do Pará, Belém - PA, Brasil.

E-mail: farp1304@gmail.com

(iD) https://orcid.org/0000-0001-9569-943X

Maély Ferreira Holanda Ramos

Professora do Programa de Pós-graduação em Educação da Universidade Federal do Pará.

Professora do Programa de Pós-graduação em Segurança Pública da Universidade Federal do Pará.

E-mail: maelyramos@hotmail.com

(iD) http://orcid.org/0000-0001-6150-6345

\section{Edson Marcos Leal Soares Ramos}

Professor do Programa de Pós-graduação em Segurança Pública da Universidade Federal do Pará, Belém PA, Brasil.

E-mail: ramosedson@gmail.com

(iD) https://orcid.org/0000-0001-5425-8531

Endereço para envio de correspondência:

Universidade Federal do Pará, Faculdade de Letras Estrangeiras Modernas (FALEM). Rua Augusto Correa, n. 1, Bairro Guamá.

Belém - Pará. Brasil.

Recebido 16/05/2017

Aceito 05/07/2018 
Psicologia: Ciência e Profissão 2019, 39, e180093, 1-16.

Received 05/16/2017

Approved 07/05/2018

Recibido 16/05/2017

Aceptado 05/07/2018

Como citar: Pereira, R. R., Silva, S. S. C., Faciola, R. A., Pontes, F. A. R., Ramos, M. F. H., Soares, E. M. L. (2019). Estresse e características resilientes em alunos com deficiência e TFE na UFPA. Psicologia: Ciência e Profissão, 39, 1-16. https://doi.org/10.1590/1982-3703003180093

How to cite: Pereira, R. R., Silva, S. S. C., Faciola, R. A., Pontes, F. A. R., Ramos, M. F. H., Soares, E. M. L. (2019). Stress and resilience characteristics of students with disability and with specific functional disorders at UFPA. Psicologia: Ciência e Profissão, 39, 1-16. https:// doi.org/10.1590/1982-3703003180093

Cómo citar: Pereira, R. R., Silva, S. S. C., Faciola, R. A., Pontes, F. A. R., Ramos, M. F. H., Soares, E. M. L. (2019). Estrés y características resilientes en alumnos con discapacidad y TFE en la UFPA Psicologia: Ciência e Profissão, 39, 1-16. https:// doi.org/10.1590/1982-3703003180093 